\title{
VALORACIÓN DEL COMPORTAMIENTO ÉTICO DE LOS RECTORES DE LAS UNIVERSIDADES DE IBEROAMÉRICA ${ }^{1}$
}

\author{
APPRAISEMENT OF TH ETHICAL BEHAVIOUR OF IBEROAMERICAN \\ UNIVERSITY RECTORS
}

FRANCISCO GANGA-CONTRERAS
Universidad de Los Lagos
fganga@ulagos.cl

GONZALO GAJARDO

Universidad de Los Lagos

ggajardo@ulagos.cl

RECIBIDO: $14 / 07 / 2016$
ACEPTADO: $04 / 08 / 2016$

Resumen: La universidad debe disponer de la capacidad administrativa y académica para monitorear y evaluar la calidad de la gestión desarrollada (gobernanza). Una encuesta realizada a expertos universitarios de 19 países de Iberoamérica (858 casos) demuestra preliminarmente que el comportamiento ético del rector es altamente valorado, pues está dotado de autoridad y se le ha conferido la responsabilidad para llevar a cabo las políticas y objetivos establecidos en el plan institucional. Su liderazgo y conducta ética son esenciales -en un ambiente complejo, dinámico y selectivo- para coordinar e integrar con responsabilidad y con visión estratégica los recursos y talentos disponibles para alcanzar óptimos resultados. Bajo esta premisa se discute el doble rol, y la opinión general de los expertos reafirma que superar este problema está relacionado con la conducta ética de la máxima autoridad universitaria. La sentencia anterior, se estructura independiente del tipo de universidad, sexo, años de servicio de los expertos y de algunas características estructurales de las universidades a las cuales pertenecen.

Palabras clave: Educación superior, ética de las organizaciones, gobierno universitario, gobernanza.

\begin{abstract}
The university must have administrative and academic capabilities to monitoring and evaluating management quality (governance). A survey responded by university experts from 19 Iberoamerican countries (858 cases) shows preliminarily the ethical behaviour of the president or principal (rector in Spanish) is highly valued since he or she has the authority, and has been given the responsibility to carry out the policies and aims established in the institutional developmental plan. Rector leadership and behaviour is essential -under a complex and dynamic and selectiveenvironment to coordinate with responsibility and strategic vision the resources and talents of the university to achieve the best possible results. Under this assumptions the double role often played by the rector in some universities is discussed. In general, experts highly value the ethical behaviour of the rector to overcome this problem. This statement is independent of the university type, sex of the
\end{abstract}

\footnotetext{
${ }^{1}$ Este trabajo es parte de los resultados preliminares del Proyecto Fondecyt Regular $\mathrm{N}^{\circ} 1161353$, por lo que los autores agradecen el apoyo y patrocinio de la Comisión Nacional de Ciencia y Tecnología de Chile (CONICYT-Chile).
} 
experts, years in the university, and of some structural characteristics of the university to which they belong.

Keywords: Higher education, organizational ethics, university government, governance

\section{Introducción}

En la sociedad del conocimiento y del manejo de la información las universidades son agentes para el crecimiento económico de los países, para la prosperidad individual y para la movilidad social. Como centros de investigación y reflexión aportan a su entorno local, nacional, regional o internacional, y tienen la responsabilidad de formar integralmente a las próximas generaciones de profesionales o líderes. Por la importancia de este rol la gobernanza universitaria surge como una conducta importante pues se refiere a la capacidad para tomar decisiones estratégicas pertinentes y sustentables en un ambiente educacional altamente dinámico y complejo, lo cual requiere igualmente disponer de mecanismos de control para monitorear y evaluar la coherencia e integridad de las decisiones adoptadas (University of Oxford 2006). La gobernanza requiere una actitud de evaluación de la gestión (accountability) de aquellos que han sido investidos con la responsabilidad y la autoridad para llevar a cabo las políticas y objetivos institucionales. Un rol esencial en este proceso le cabe a los cuerpos colegiados y a la comunidad académica, pero la figura del rector es relevante para el éxito, calidad y devenir del proyecto institucional, puesto que sus características académicas, éticas, de liderazgo y de personalidad (King 2008) son importantes para la estabilidad de una organización jerarquizada de acuerdo al mérito, pero también pues el rector es la cara visible y el reflejo del proyecto institucional que encabeza ante la comunidad. En un escenario social y educacional en constante evolución el proyecto institucional debe ser revisado permanentemente. El rector tiene capacidad para decidir múltiples cuestiones, dependiendo de la estructura de poder establecida en los estatutos. Por ejemplo, hay cuerpos colegiados-consultivos en donde el rector sólo puede opinar, mientras que en otros el rector preside y decide las temáticas a discutir y muchas veces puede condicionar las decisiones del cuerpo colegiado, lo cual puede ocurrir en una estructura piramidal de poder, con poco control. Ello puede ser o no conveniente dado el ámbito de cuestiones en las que el rector puede decidir: el tipo de investigación que la universidad realiza (básica, aplicada, en colaboración con la empresa), lo cual puede significar privilegiar a ciertos grupos por sobre otros. El rector puede igualmente influir en la estructura interna de gestión, en las carreras impartidas, el acceso y admisión, puede otorgar asignaciones económicas discrecionales y de esta forma desvirtuar la jerarquía académica, y 
de manera cada vez creciente el rector tiene que tomar decisiones con la relación a la industria que demanda servicios a la universidad.

Ahora bien, en muchas universidades existe aún la tentación de combinar en una sola persona o cargo, las funciones contraloras y las de dirección ejecutiva y estratégica. Evidentemente en estas circunstancias se concentra mucho poder en el directivo superior, fenómeno que la literatura le ha denominado "conflicto de doble rol”. En este sentido, lo lógico sería que el rector no presidiera el máximo cuerpo colegiado.

Asumiendo que el rector es el reflejo del proyecto universitario y que su liderazgo es importante para la toma de decisiones estratégicas adecuadas y para integrar adecuadamente la diversidad de capacidades humanas disponibles en la universidad, este artículo analiza la opinión que expertos de diferentes universidades Iberoamericanas tienen sobre la importancia de la ética y liderazgo del rector en relación a un tema tan complejo como podría ser el conflicto de doble rol.

El artículo es una inquietud preambular que surge de un estudio más amplio sobre gobernanza universitaria. Se presentan inicialmente algunos elementos de contexto tomados desde la biología, pues se asume que una organización como la universidad que está compuesta por seres humanos, responde en parte a la lógica de los seres vivos. Luego se informa la metodología referente a la encuesta que hace posible este análisis preliminar y los resultados que confirman que la inquietud sobre el rol del rector es generalizada en los encuestados, cualquiera sea la variable analizada, sea: sexo, cargo y grado académico de los encuestados.

\section{Fundamentos teóricos}

\section{Seres vivos y organización}

La premisa de este artículo es que las organizaciones humanas son sistemas complejos, que evolucionan gracias al potencial de cambio que otorga la gran diversidad de características que son inherentes a los sistemas biológicos, desde el nivel molecular, fisiológico al fenotípico externo (conducta, liderazgo, honestidad, altruismo, confiabilidad, entre otros). La expresión de estos rasgos depende de la interacción entre factores biológicos (genética que integra igualmente la historia de la especie) y ambientales. Darwin en su libro Origen de las Especies estableció que la persistencia en el tiempo del mundo viviente, desde las bacterias a los humanos, dependía de leyes naturales como la Selección Natural actuando sobre la diversidad de características que cada 
organismo exhibe. Aquellos individuos con variantes ventajosas para resistir las presiones selectivas locales sobreviven (reproducción diferencial) mientras que individuos con características menos ventajosas tendían a desparecer o disminuir en frecuencia. Maturana y Varela (1980) igualmente describieron en su concepto de autopoiesis una característica esencial que distingue a lo vivo de lo no vivo y que es la capacidad de auto-regularse y autogenerarse a través de la interacción de sus componentes.

Con las precisiones que corresponda pensamos, como se explicará con algún detalle posteriormente, que las universidades como entidades compuestas por humanos son regidas por las mismas leyes naturales o intrínsecas que rigen a los seres vivos, con la diferencia del factor cognitivo. En otras palabras, la universidad evoluciona en el tiempo hacia estados superiores de organización producto de las propiedades cognitivas de sus componentes ("inteligencia organizacional"), y gracias a la capacidad de memoria que trasciende a los individuos que las componen en un determinado momento de la historia organizacional. La diversidad asegura la permanencia en el tiempo de un proyecto institucional coherente que tiene como elemento unificador el mérito, cuya expresión es la organización jerárquica. Asimismo, la búsqueda de la verdad y la reflexión metódica en el quehacer investigativo, todo lo cual tributa a la formación integral de los recursos humanos y a la solución de los problemas que aquejan a la comunidad, uno de los más importantes es la inequidad en el acceso a una educación de calidad. Hay diversidad de talentos, cultores de diferentes disciplinas y artes y una diversidad de conductas en la universidad, de aquí que la ética, liderazgo y personalidad del rector son fundamentales para integrar la misión institucional en un todo coherente con una visión colectiva y altruista (Ciulla 2014). En un ambiente cambiante o incierto para las universidades, el liderazgo ético tiene que ver con libertad, trascendencia, congruencia en los valores, acción cooperativa, poder, persuasión y gobernanza corporativa. En este sentido, podría aducirse que mientras la ética es una cualidad sustentada en principios que idealmente son permanentes, las características del líder están en permanente evolución y es deseable que su conducta ética no mute de acuerdo a las circunstancias, y para ello la autonomía y capacidad reflexiva de los órganos colegiados que representan a los académicos y a la sociedad son esenciales, especialmente en universidades de carácter público que se deben a la comunidad. En este contexto las decisiones estratégicas deben ser coherentes, transparentes y evaluables ("accountability") para no afectar la viabilidad del proyecto organizacional. El líder narcisista preocupado de acciones que privilegian su interés personal es la versión negativa de lo que la universidad requiere puesto que la fortaleza institucional reside, 
como se dijo, en la diversidad y en las redes de colaboración que se establezcan tanto al interior como en el entorno nacional o internacional. Adquiere así relevancia el concepto de autopiesis de Maturana y Varela que ha sido extrapolado para entender el funcionamiento de los sistemas sociales y organizacionales, que son entidades más complejas (Hall 2003; Goldspink y Kay 2007). La autopoiesis apunta literalmente a una de las propiedades más distintivas de los seres vivos que es la auto-organización, es decir, la dinámica de sus componentes colectivamente produce y mantiene la organización del sistema. Esto tiene profundo significado para el quehacer y calidad de un determinado proyecto universitario. Otras propiedades distintivas de los seres vivos que se aplican a una universidad son: 1) tener límites que lo identifiquen como unidad autónoma y que lo separan del medio externo, es decir la identidad o misión de una universidad es esencial; 2) el conjunto de componentes (autoridades, cuerpos colegiados, académicos) identifican al todo; 3) las propiedades del sistema son generadas por la interacción de sus componentes y no son simplemente la suma de las propiedades de cada componente individual. Entonces la pregunta esencial es como la universidad crea el conocimiento y la memoria necesaria para la replicación de sus propiedades en el tiempo. Ello inexorablemente está ligado a la estructura y funcionamiento de la organización y recae en características que son innatas a la condición animal (verbi gratia: egoísmo, haraganería, agresividad, altruismo, cooperación, etc) pero que han sido moldeadas por el entorno familiar y educacional (ambiente). De aquí que las características conductuales (liderazgo) y éticas del Rector tengan finalmente relevancia para la visualización e impacto del proyecto institucional que encabeza, y para que este proyecto alcance niveles superiores de organización. La manera de medir este progreso son actualmente los rankings universitarios que sirven el propósito de informar la comunidad cuales son los mejores proyectos universitarios sobre la base de definir determinadas variables comparables. Uno de estas es el prestigio y como se ha indicado, el rector es la cara visible de la reputación universitaria.

\section{Gobernanza universitaria y conflicto de doble rol}

Una de las cuestiones relevantes que están asumiendo los investigadores del campo de la educación superior, dice relación con lo que ha venido llamándose la gobernanza de las universidades, la cual comprende una serie de componentes entre los cuales se pueden destacar: elementos externos como son los órganos respectivos del Estado (gobierno nacional, ministerios, parlamento, poder judicial, agencias públicas especializadas) y la sociedad civil 
(empresarios, asociaciones y organizaciones sociales, profesiones y graduados, opinión pública); elementos internos de las propias corporaciones académicas, como docentes e investigadores, estudiantes, trabajadores administrativos y técnicos; diversos tipos de instituciones que integran el sistema, como universidades docentes, complejas, semi-complejas y unidades funcionales, como facultades, institutos, departamentos, carreras, centros, etc. y; formas de gobierno, liderazgos, estructuras y procedimientos, políticas organizacionales (académicas e institucionales), planes estratégicos así como procesos decisionales (Ganga, Abello y Quiroz 2014, Ganga, Fossatti y Quiroz 2016, Brunner y Ganga 2016, Brunner y Ganga 2016 b).

Respecto del último elemento precitado, una de las materias complejas que se abordan en los códigos de buen gobierno organizacional, dice relación con la separación entre dirección y control; lo anterior debido a que en muchas instituciones, el máximo ejecutivo -además de tener la responsabilidad de la dirección general de la respectiva entidad- asume también la función de presidir el cuerpo colegiado superior -el cual debe monitorear la labor del directivogenerando con ello, lo que se conoce como conflicto de doble rol. Es por lo anterior, que existen una buena cantidad de autores que sostienen que es ineluctable lograr la separación de ambas tareas, dado que, de esta forma, se reduce el poder del principal directivo y junto con ello, se acrecienta la capacidad supervisora del máximo cuerpo colegiado (Patton y Baker 1987, Termes 1998, Olivencia 1998, Galve 2002, Martínez 2003, Mínguez y Martín 2005, Stein y Capape 2009 y Cazorla 2012).

\section{Antecedentes metodológicos}

Se pretende analizar la opinión que tienes los expertos de universidades de Iberoamérica, respecto de la importancia de la ética del Rector, en el contexto del gobierno universitario. Pregunta que se enmarca en una investigación mayor, acerca de la gobernanza universitaria.

Para alcanzar la finalidad previamente descrita, se procedió a la aplicación de un cuestionario, a una muestra intencionada, que permitiera la recolección de opiniones de la máxima cantidad de expertos posibles, de diversos países y cargos universitarios. Para efectos de este trabajo, se entiende por expertos a personas que ocupen o hayan ocupado cargos de gestión: jefes de área, carrera y/o programas, directores, decanos, vicerrectores y rectores; también se consideraron académicos y profesionales que estudien, tengan experiencia o conozcan los temas de gobierno organizacional o sistemas universitarios. 
El instrumento de recolección de información se validó considerando en primera instancia la técnica "validación de expertos" y de un "pretest cognitivo"; luego de la etapa anterior, se aplicó el cuestionario, enviándose por correo electrónico y/o presencialmente en los diversos congresos o encuentros de expertos sobre la materia.

La base final quedó constituida por una muestra de 858 expertos de 19 países iberoamericanos, de un conjunto de 220 Universidades. El número de respuestas por países, puede observarse en la figura $\mathrm{N}^{\circ} 1$.

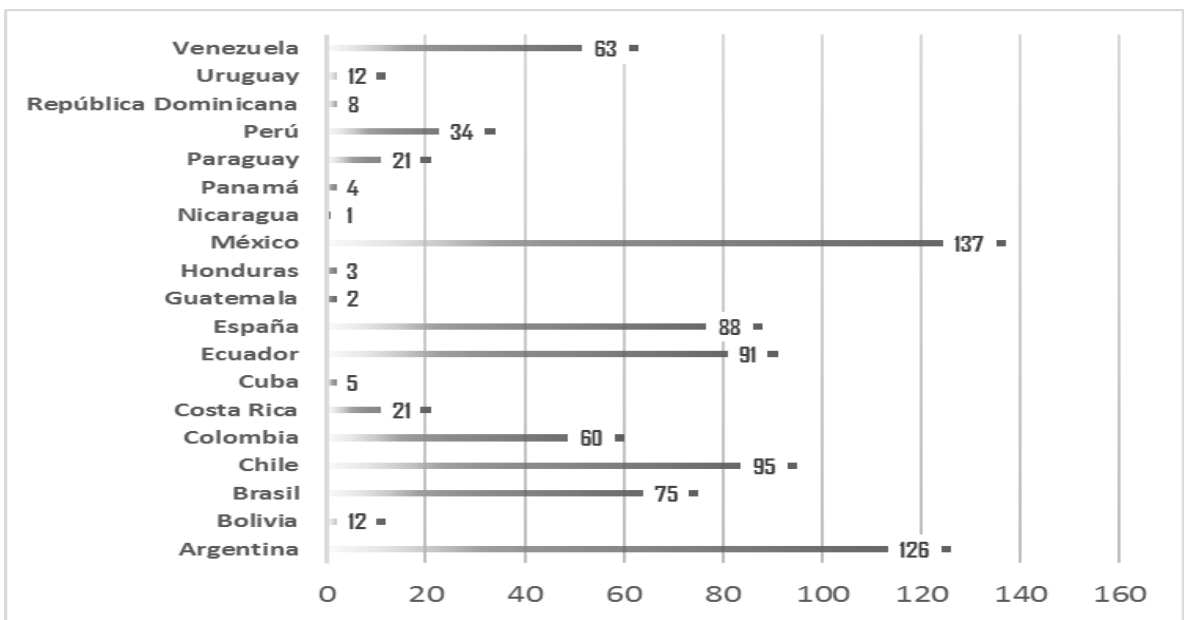

Figura 1. Respuestas de expertos por países. Fuente: Elaboración propia, Encuesta Expertos Gobernanza Universitaria

\section{Presentación de resultados}

\section{Importancia del comportamiento ético del máximo directivo}

El rol del Rector es innegable por lo señalado precedentemente, como centro y articulador de la gestión de la Universidad. Independiente de las configuraciones estructurales de la Universidad, o de la serie de atribuciones que pueda poseer, la forma en que se desenvuelve y da uso a esas atribuciones e instancias definen el tipo de relación que tendrá con el resto de los estamentos de la institución.

En este contexto, se les plantea a los expertos de Iberoamérica que opinen en relación al grado de acuerdo que tienen respecto a la afirmación: "independiente 
del problema del doble rol, lo más relevante es el comportamiento ético del rector."

Como se puede observar en la figura $\mathrm{N}^{\circ} 2$, sobre el $85 \%$ de los encuestados se mostró concordante con la afirmación de que independiente del problema de doble rol, lo más relevante es el comportamiento ético de la máxima autoridad universitaria.

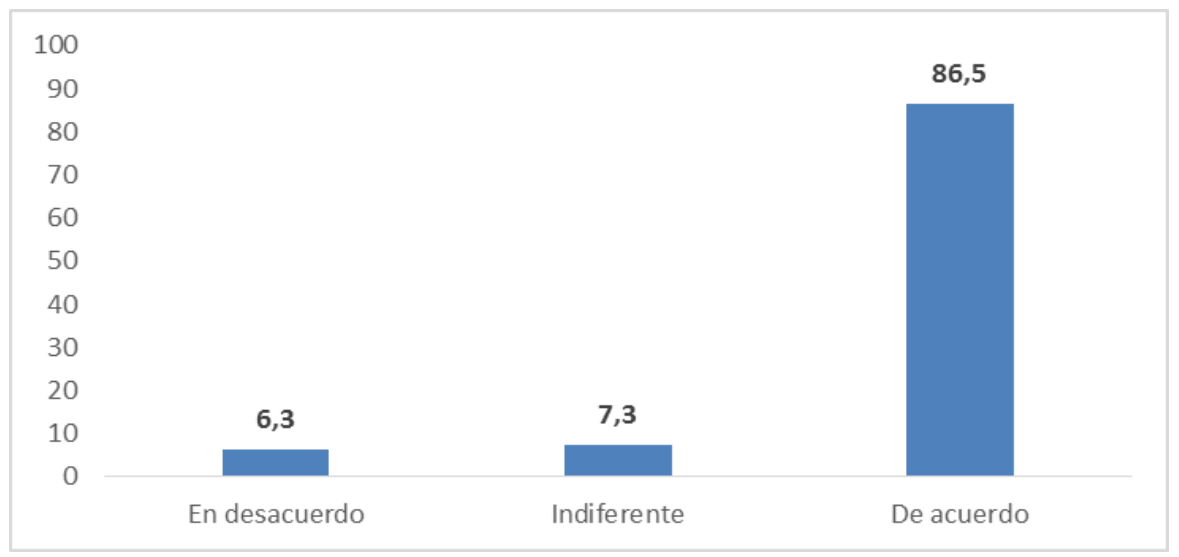

Figura $\mathrm{N}^{\circ}$ 2. Independientemente del problema de doble rol, lo más importante es el comportamiento ético del Rector. Fuente: Diseño propio, basado en análisis estadístico de las encuestas recibidas

Opinión en función de las características de los encuestados

En el afán de abrir el resultado de esta consulta inicial, se cruzaron estas opiniones con una serie de características relevantes de los expertos (as) consultadas (as), entre los que se encuentran: el cargo en la universidad, el grado académico, años que lleva trabajando en la universidad y el sexo. El cargo en la universidad es relevante, en la lógica de que podrían existir diferencias de opinión, según las tareas y acceso a responsabilidades en la universidad de los expertos que opinan. Es de esta manera que los cargos se agruparon en tres grandes grupos, en función de la cercanía que este tiene a las instancias de toma de decisión dentro de la Universidad, quedando como sigue:

a) Altas autoridades, el cual está compuesto por rectores, ex-rectores, vicerrectores y decanos. 
b) Jefaturas intermedias, compuesto básicamente por jefes de departamento y posiciones afines, $y$

c) Profesores e investigadores de las universidades.

Lo que se observa como respuesta a la consulta es transversal al cargo universitario del experto encuestado, ya que no se identificaron diferencias estadísticamente significativas entre ellos. Todos consideran que una estrategia viable para superar los posibles problemas de doble rol en la Universidad, es que el rector posea un comportamiento ético que permita encontrar soluciones que superen los peligros del autoritarismo (ver figura $\mathrm{N}^{\circ} 3$ ).

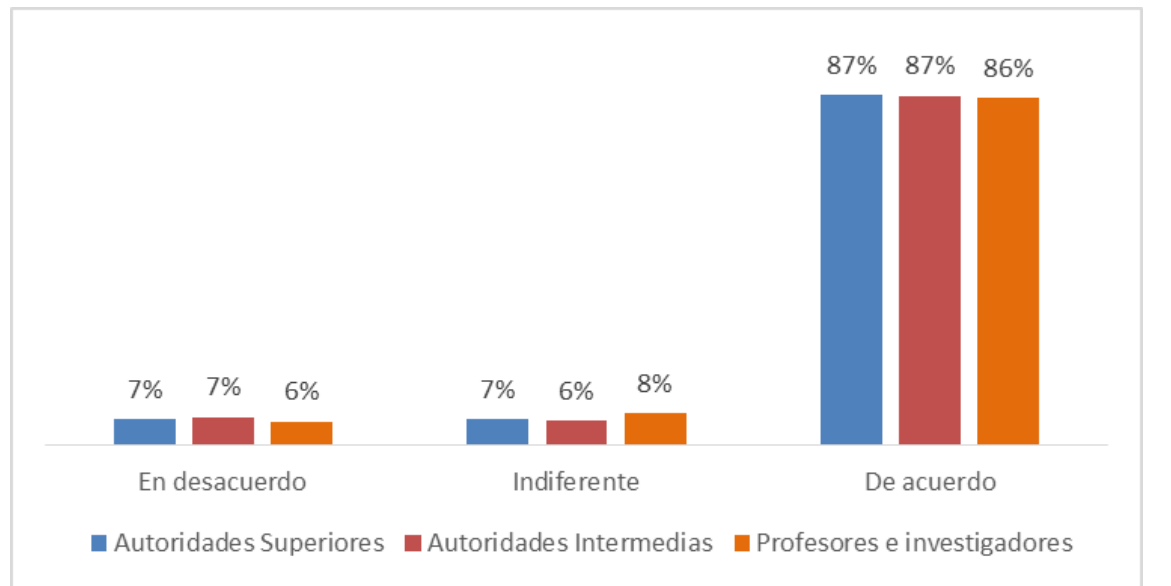

Figura 3. Distribución según cargo universitario para la afirmación: Independientemente del problema de doble rol, lo más importante es el comportamiento ético del Rector. Fuente: Diseño propio, basado en análisis estadístico de las encuestas recibidas

Los expertos encuestados fueron claros en señalar la existencia de posible conflicto de doble rol, cuando el rector participa con plenos derechos en las instancias del Máximo Cuerpo Colegiado, el cual se puede subsanar planteando soluciones organizativas de estas instancias, para minimizar la presencia de este problema.

Aun así, consideran que el comportamiento ético del rector, es una de las mejores bazas que puede solucionar o evitar que se desarrollen estos problemas, en función del liderazgo que pueda ejercer para implementar soluciones compartidas. 
Al revisar las respuestas según el grado académico del encuestado, vemos que estas no son independientes, ya que al realizar la prueba de Chi-cuadrado se obtuvo resultados estadísticamente significativos ${ }^{2}$. De esta manera se percibe que los expertos que tienen Magister o Master concentran su opinión ${ }^{3}$ a estar de acuerdo con la importancia de la ética del Rector para lidiar con los problemas asociados al doble Rol. Mientras que los expertos con solo Licenciatura, que en su mayoría están de acuerdo con la afirmación, presenta un porcentaje importante de estar indiferentes a esta afirmación (ver figura $\mathrm{N}^{\circ} 4$ ).

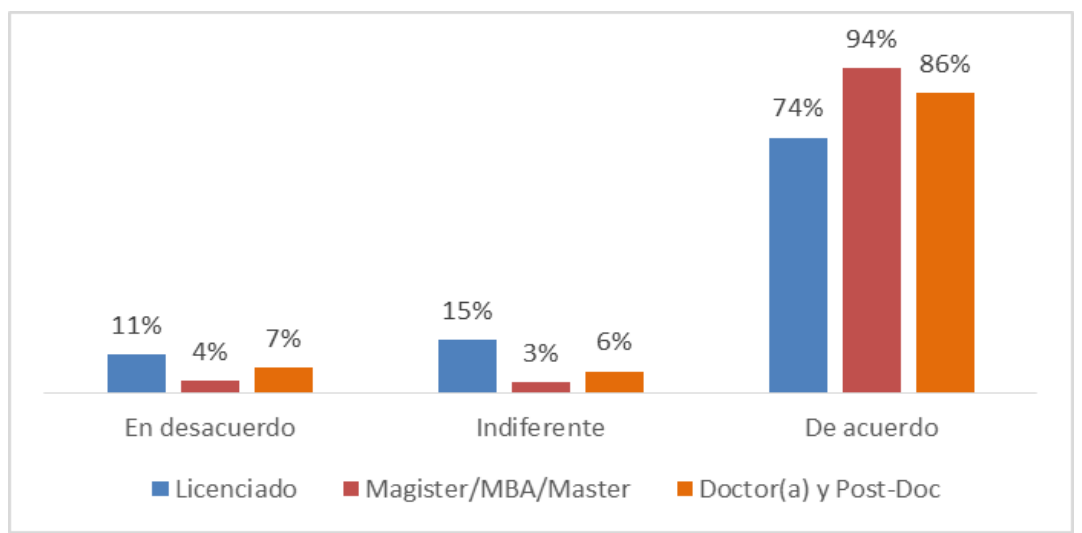

Figura $\mathrm{N}^{\circ}$ 4. Distribución según grado académico para la afirmación: Independientemente del problema de doble rol, lo más importante es el comportamiento ético del Rector. Fuente: Diseño propio, basado en análisis estadístico de las encuestas recibidas

Al revisar la variable años en la universidad del experto, vemos que presenta una asociación significativa cuando realizamos la prueba de Chi-Cuadrado, con las opiniones acerca de la importancia de la ética del Rector. De este modo, como se observa en la Figura 5, los expertos que poseen menos de un quinquenio en la institución educativa son los que concentran significativamente las opiniones de

\footnotetext{
${ }^{2}$ La prueba de Chi-Cuadrado es un estadístico que permite probar la asociación entre dos variables. Donde el valor de significación de la prueba debe ser menor a 0,05 para rechazar la hipótesis de independencia entre las dos variables.

${ }^{3}$ Para identificar la concentración de opiniones, se realizó un análisis de residuos tipificados o corregidos, el cual nos permite, a un nivel de confianza de 0,95 , que los valores de residuos mayores a 1,96 delatan casillas con más casos de los que debería haber en esa casilla si las variables estudiadas fueran independientes.
} 
"estar en desacuerdo" con la respectiva aseveración; mientras que los expertos que tienen entre 13 a 20 años de vida laboral en las universidades, son los que no le dan mayor importancia del comportamiento ético del rector (ver figura $\mathrm{N}^{\circ} 5$ ).

\begin{tabular}{|c|c|c|c|c|c|c|c|}
\hline & & $\begin{array}{c}\text { Menos de } 1 \\
\text { a } 5 \text { años en } \\
\text { universidad }\end{array}$ & $\begin{array}{c}6 \text { a } 12 \text { años } \\
\text { en la } \\
\text { universidad } \\
\end{array}$ & $\begin{array}{c}13 \text { a } 20 \\
\text { años en la } \\
\text { universidad } \\
\end{array}$ & $\begin{array}{c}21 \text { a } 30 \\
\text { años en la } \\
\text { universidad }\end{array}$ & $\begin{array}{c}\text { Más de } 30 \\
\text { años en la } \\
\text { universidad }\end{array}$ & Total \\
\hline \multirow[t]{3}{*}{$\begin{array}{l}\text { En } \\
\text { desacuerdo }\end{array}$} & Recuento & 15 & 12 & 4 & 9 & 4 & 44 \\
\hline & $\begin{array}{l}\text { Frecuencia } \\
\text { esperada }\end{array}$ & 9,5 & 8,2 & 12,3 & 10,3 & 3,7 & 44 \\
\hline & $\begin{array}{l}\text { Residuo } \\
\text { corregido }\end{array}$ & 2,1 & 1,5 & $-2,9$ & $-0,5$ & 0,1 & \\
\hline \multirow[t]{3}{*}{$\begin{array}{l}\text { De } \\
\text { acuerdo }\end{array}$} & $\begin{array}{l}\text { Recuento } \\
\text { Erecuencia }\end{array}$ & 125 & 112 & 184 & 151 & 52 & 624 \\
\hline & esperada & 135,1 & 115,9 & 174,3 & 145,5 & 53,2 & 624 \\
\hline & $\begin{array}{l}\text { Residuo } \\
\text { corregido }\end{array}$ & $-2,7$ & $-1,1$ & 2,4 & 1,4 & $-0,5$ & \\
\hline \multirow[t]{3}{*}{ Indiferente } & Recuento & 15 & 9 & 12 & 7 & 5 & 48 \\
\hline & $\begin{array}{l}\text { Frecuencia } \\
\text { esperada }\end{array}$ & 10,4 & 8,9 & 13,4 & 11,2 & 4,1 & 48 \\
\hline & $\begin{array}{l}\text { Residuo } \\
\text { corregido }\end{array}$ & 1,7 & 0 & $-0,5$ & $-1,5$ & 0,5 & \\
\hline \multirow[t]{2}{*}{ Total } & Recuento & 155 & 133 & 200 & 167 & 61 & 716 \\
\hline & $\begin{array}{l}\text { Frecuencia } \\
\text { esperada }\end{array}$ & 155 & 133 & 200 & 167 & 61 & 716 \\
\hline
\end{tabular}

Figura 5. Cruce Independiente del problema de doble rol con los años en la Universidad del experto encuestado. Detalle de los valores del análisis de residuos Fuente: Diseño propio, basado en análisis estadístico de las encuestas recibidas

Adicionalmente, en relación al sexo de los encuestados, de la figura 6 se desprende que la opción predominante es la de estar de acuerdo con la afirmación. Si bien para las mujeres el porcentaje en desacuerdo es mayor que el de los hombres, esta diferencia no es estadísticamente significativa, como para plantear la existencia de relación entre las dos. 


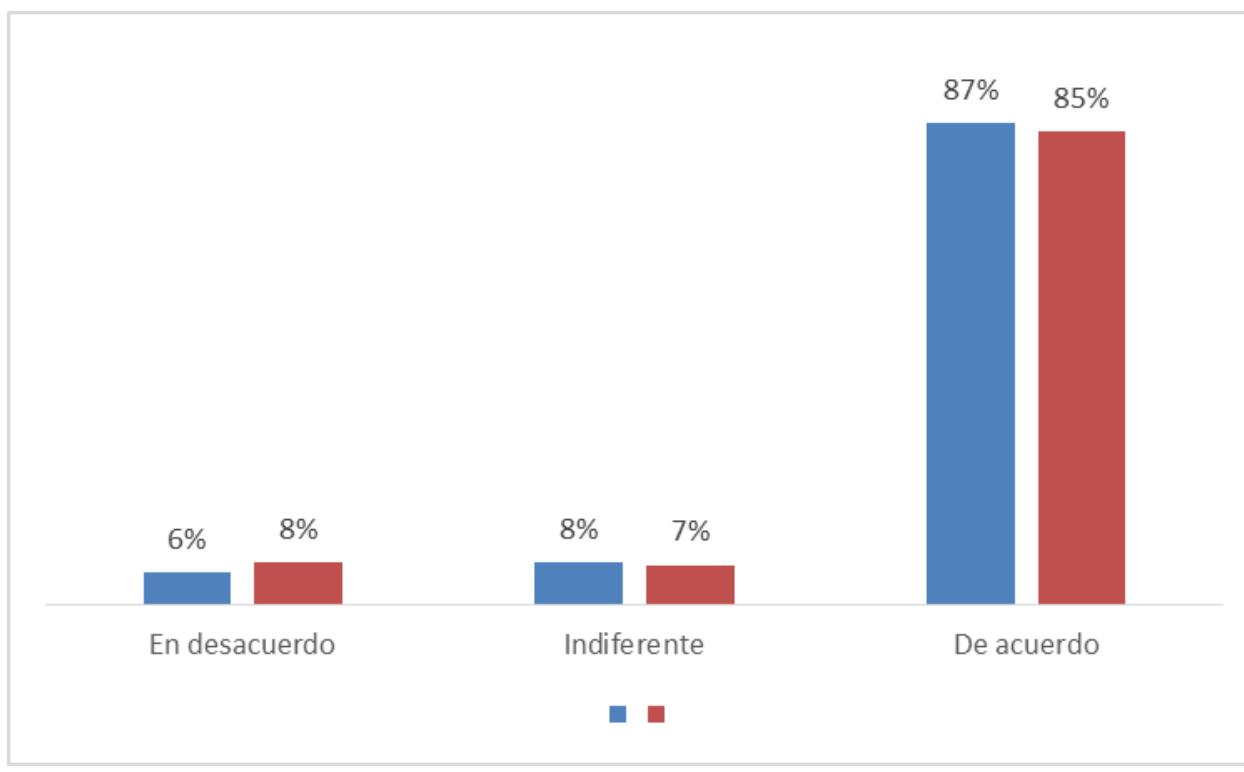

Figura 6. Distribución según sexo para la afirmación: Independientemente del problema de doble rol, lo más importante es el comportamiento ético del Rector. Fuente: Diseño propio, basado en análisis estadístico de las encuestas recibidas

En resumen, y habiendo cubierto las principales características de los expertos que perfilan su relación con las instituciones universitarias, vemos que la opinión es general en cuanto a considerar a la ética del Rector como la mejor herramienta para dar solución a los problemas derivados del posible conflicto de doble rol, que puede operar en su institución. Esta certeza puede no estar totalmente afincada en los expertos que llevan poco tiempo trabajando en la universidad, pero que es transversal al resto de los estamentos. Esta postura no es menor, teniendo en cuenta la diversidad de estructuras, organigramas y configuraciones de responsabilidad que tienen las distintas universidades de Iberoamérica.

Apreciaciones sobre el comportamiento ético según características de la Universidad

Una vez revisadas las características asociadas a los expertos, se trabajó con las variables que perfilan a las universidades, para observar cómo se comporta o varían las opiniones acerca de la importancia de la ética del Rector, en esas 
instituciones. Entre las variables a analizar se encuentran: país de la universidad, tipo de universidad, quien es el que preside el máximo cuerpo colegiado y el tipo de elección de la máxima autoridad.

Como se observa en la Figura 7, la opinión acerca de la importancia de la ética del Rector es transversal a los países ${ }^{4}$, ya que no se obtuvieron diferencias estadísticamente significativas entre ellos. Aun así, es posible identificar ciertos países donde el disenso de la importancia de la ética es más marcado, pero no logran revertir la tendencia general de aprobación.

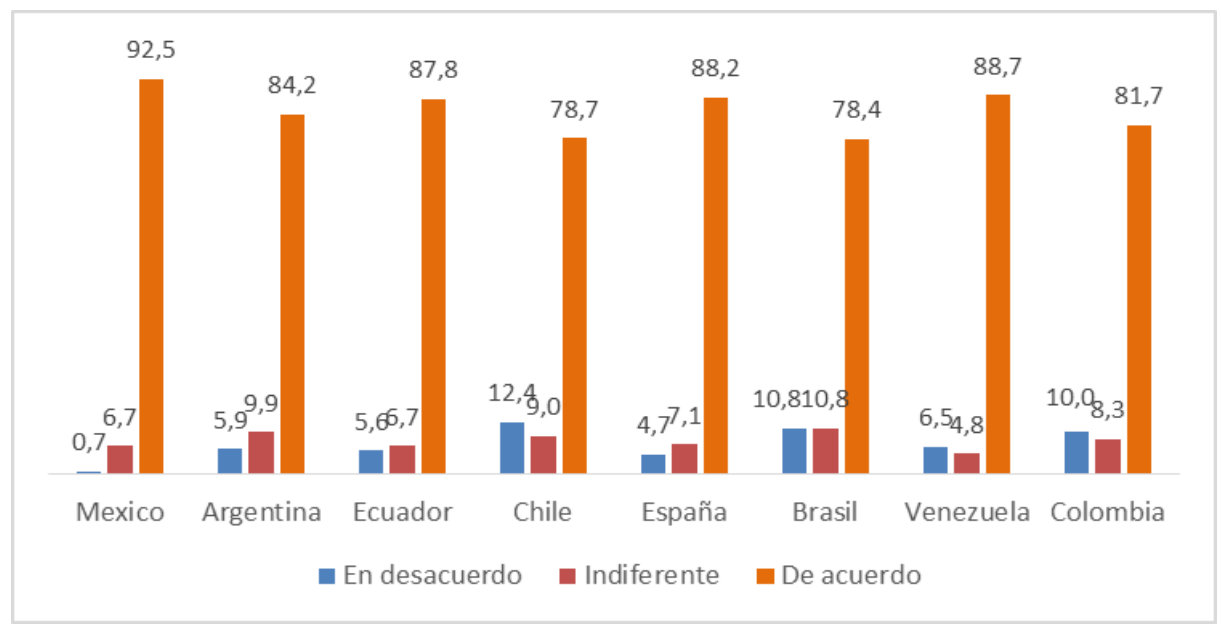

Figura 7. Distribución según país para la afirmación: Independientemente del problema de doble rol, lo más importante es el comportamiento ético del Rector. Fuente: Diseño propio, basado en análisis estadístico de las encuestas recibidas

Al revisar las opiniones según el tipo de dependencia de la Universidad (pública, privada o confesional), podemos observar en la figura 8, que la posición afirmativa a la importancia de la ética del rector, es la mayoría en cada tipo de universidad. Si bien las universidades de tipo confesional aparecen con un porcentaje mayor de aprobación, las diferencias no son estadísticamente significativas, es decir no hay asociación entre la respuesta y el tipo de universidad.

\footnotetext{
${ }^{4}$ En el cuadro están presentes los países que contabilizaban más de 50 casos en la base de datos.
} 


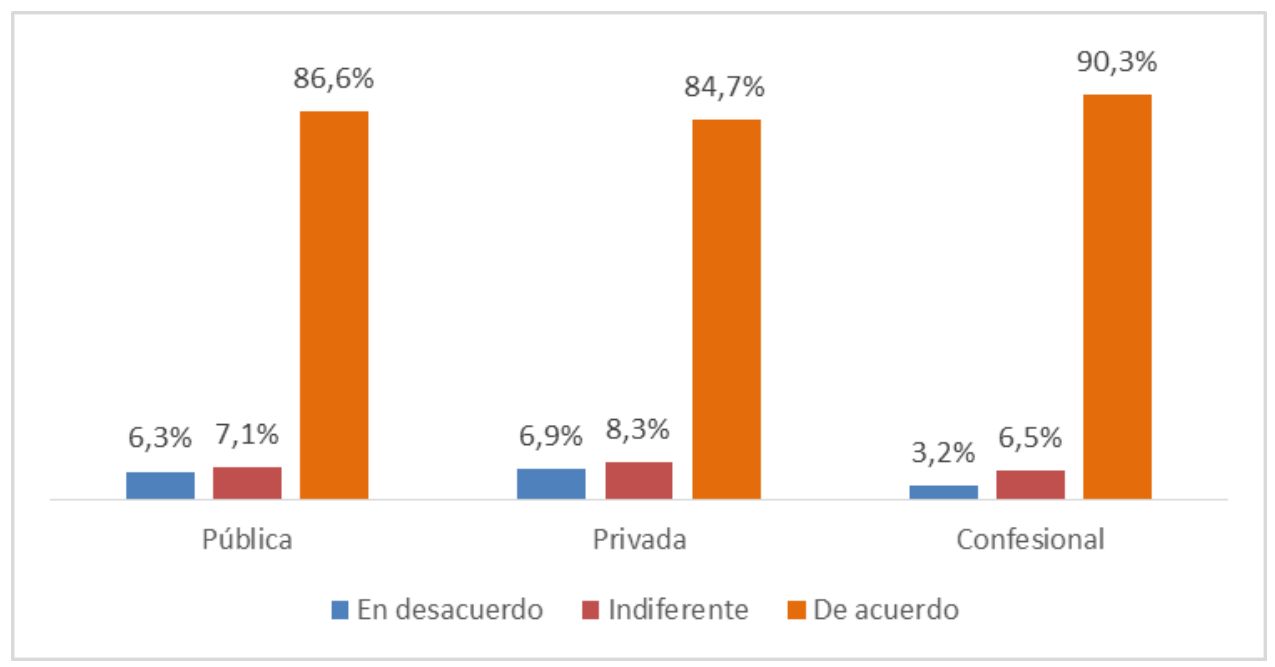

Figura 8. Distribución según tipo de Universidad para la afirmación: Independientemente del problema de doble rol, lo más importante es el comportamiento ético del Rector. Fuente: Diseño propio, basado en análisis estadístico de las encuestas recibidas

Otra característica de la universidad que se revisó fue identificarlas según quién es el presidente del Máximo Cuerpo Colegiado. De esta manera se ordenaron según las siguientes opciones: El Rector, un integrante elegido por los miembros del MCC, el integrante más antiguo del MCC u otra persona.

Como se observa en la Figura $\mathrm{N}^{\circ}$ 9, independiente de quien dirige el MCC, existe una posición transversal para considerar que lo más importante es la ética del Rector.

Si bien no existen diferencias estadísticamente significativas entre estas dos variables, por lo que no podemos hablar de asociación, aun así, se observa que en el caso de los MCC presididos por uno de sus integrantes, aumentan las opiniones desfavorables a la importancia de la ética del Rector. Pero esta diferencia no es suficiente para marcar una tendencia. 


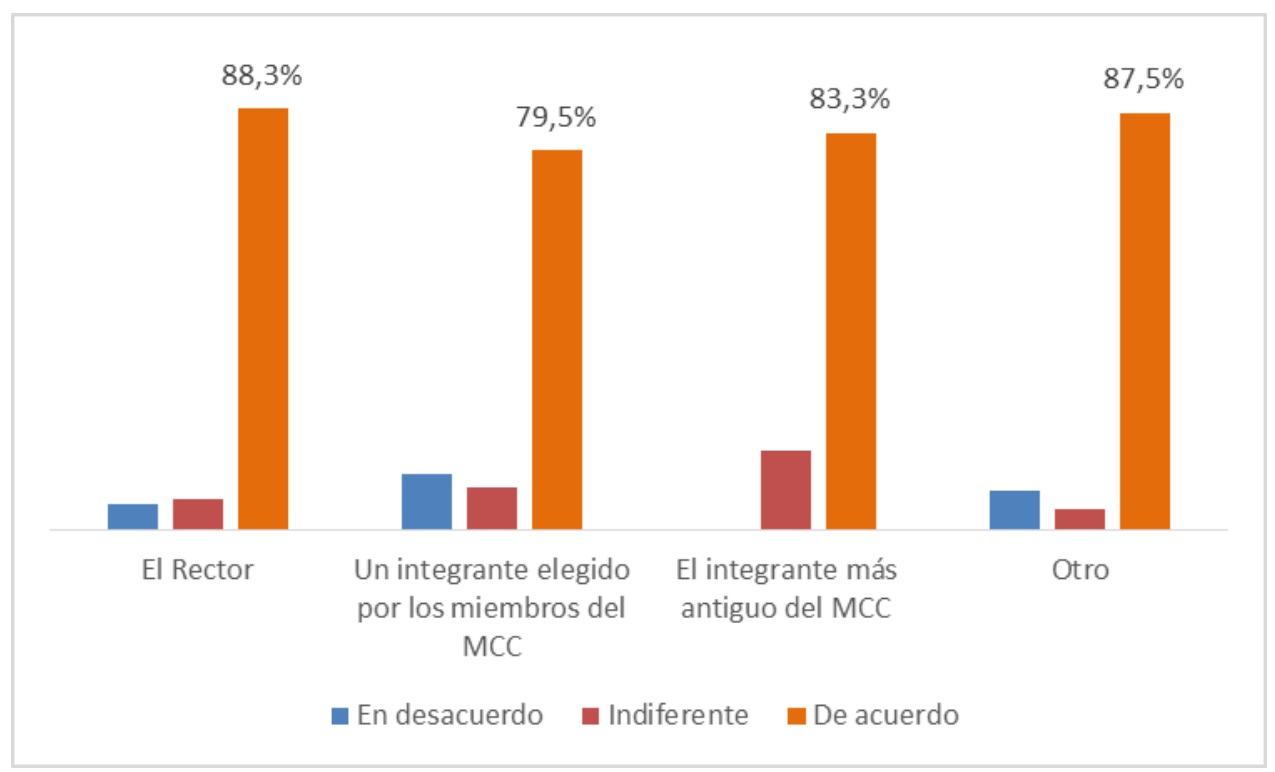

Figura 9. Distribución según quien preside el máximo cuerpo colegiado para la afirmación: Independientemente del problema de doble rol, lo más importante es el comportamiento ético del Rector. Fuente: Diseño propio, basado en análisis estadístico de las encuestas recibidas

Finalmente, se revisó la opinión de los expertos según el tipo de elección de la máxima autoridad universitaria. Este análisis se realiza, dado que en muchas universidades el rector es elegido por votación (un académico un voto) lo que desvirtúa el principio de jerarquía que es pilar de una universidad saludable. Es por ello que universidades de alto prestigio utilizan mecanismos diferentes para la búsqueda del rector pensando, precisamente, en la calidad del elegido.

Para efectos de presentar resultados, las universidades se agruparon en aquellas en que: i) todos los estamentos de la universidad tienen derecho a voto; ii) el máximo cuerpo colegiado tiene derecho a voto, ante una terna propuesta por un comité de expertos; iii) solo los pares académicos tienen derecho a voto u iv) otra forma de elección.

Como se puede apreciar en la figura $\mathrm{N}^{\circ} 10$ se mantiene la tendencia transversal de estar de acuerdo con que la ética del Rector es lo más importante. $\mathrm{Si}$ bien podemos observar algunas diferencias en la crítica en universidades 
donde solo votan los pares académicos, esta no es significativa para hablar de asociación.

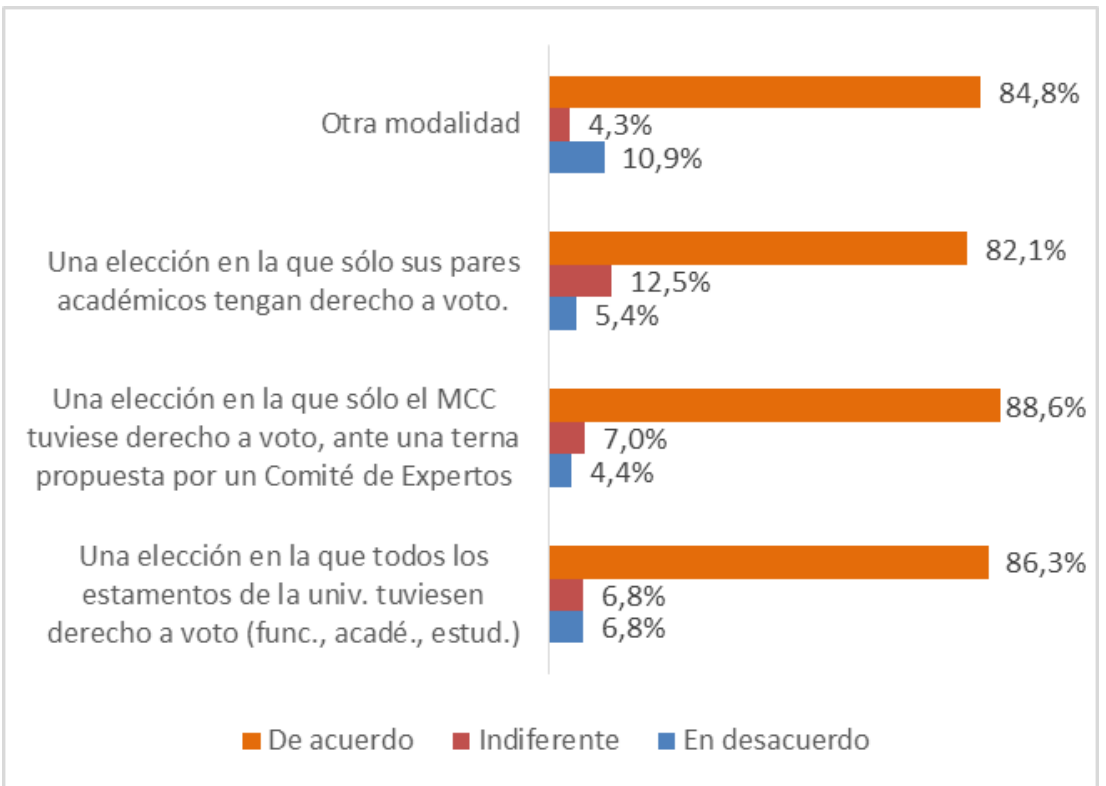

Figura 10. Distribución según tipo de elección de la máxima autoridad universitaria para la afirmación: Independientemente del problema de doble rol, lo más importante es el comportamiento ético del Rector. Fuente: Diseño propio, basado en análisis estadístico de las encuestas recibidas

En resumen, y habiendo revisado algunas de las características que perfilan a las distintas universidades, se observa una posición general de aprobación hacia la postura de la importancia de la ética del Rector. Esto ocurre de manera independiente de que algunos casos, como en la composición del MCC, se vislumbren diferencias, aunque no significativas, pero que podrían indicar un ámbito donde se requiere más herramientas para solucionar el problema del doble rol, y no solo confiar en las capacidades éticas del rector. 


\section{Conclusiones}

1) La calidad de un proyecto universitario depende, como en toda organización compuesta de seres vivos, de su historia (tradición), de la calidad de sus componentes (directivos y académicos), de sus inter-relaciones internas y externas, y especialmente, de la conducta ética de sus miembros y autoridades, para tomar decisiones estratégicas razonadas y adecuadas al entorno presente y futuro. El éxito de esta gestión dependerá, a su vez, del filtro que ejercen los cuerpos colegiados, que debieran ser los encargados de valorar y velar por un adecuado gobierno institucional. Pero es innegable que el camino para llegar a estas metas, está plagado de óbices, muchos de los cuales se los autoimpone la propia entidad; tal es el caso de la figura que tienen muchas universidades, que implica que el directivo superior debe asumir por estatuto, de manera concomitante el papel de presidente de su respectivo máximo cuerpo colegiado. Un escenario como éste conduce inevitablemente a una concentración excesiva de poder en un solo sujeto.

2) La opinión general de los expertos con respecto a la mejor estrategia para superar la presencia del problema de doble rol en la gestión y dirección de la universidad, pasa por la conducta y ética de la máxima autoridad universitaria. La sentencia anterior, se estructura independiente del tipo de universidad, sexo, años de servicio y de algunas de sus características estructurales. En todos los casos la importancia de la ética es también transversal a las características personales de ellos.

3) Los expertos consideran, independientemente de las soluciones o mecanismos estructurales que se puedan implementar en las universidades para minimizar los efectos y problemas asociados a la supervisión de las decisiones de gestión de la máxima autoridad, que la existencia de una especie de código de conducta debe regir a la máxima autoridad. Dicho en términos aún más concretos, el trabajo deja en evidencia el hecho cierto de que si en alguna universidad existen las condiciones reglamentarias, vacíos o caquexias procedimentales, burocráticas y/o jurídicas, que generan condiciones reales para la emergencia de comportamientos oportunistas o narcisistas, problemas de riesgo moral (en la lógica de la teoría de agencia) y eventualmente actitudes y autoritarias de los máximos directivos, lo único que puede aminorar esa probabilidad, son los valores y la ética del sujeto que asume el rol de rector. Sin que se hubiera consultado, agregamos que la conducta ética de los académicos es igualmente un activo del patrimonio institucional. Esta conducta es, además, inherente a una institución que genera conocimiento a partir de la observación de la realidad. 
4) Con estas opiniones los expertos construyen el perfil que debería poseer la máxima autoridad, con características personales que puedan funcionar independiente de las variadas realidades de las universidades iberoamericanas.

Se entiende que este estudio es de carácter preliminar, pero aun cuando se reconocen las limitaciones inherentes de una encuesta, la información recolectada permite definir futuros acercamientos y profundizaciones de un tema que es vital para la gestión universitaria, que es la definición del perfil y características que debería poseer la máxima autoridad de la universidad.

\section{Bibliografía}

Brunner, J. y Ganga, F. (2016). Reflexiones en torno a economía política y gobernanza de los sistemas nacionales e instituciones de educación superior en América Latina. Revista Interciencia. Vol. 41. N 8: 573-579.

Brunner, J. y Ganga, F. (2016 b). Gobernanza de la educación superior: una mirada crítica y propositiva al caso de Chile. Revista Dilemas Contemporáneos: Educación, Política y Valores. Año: IV. Número: 1. Artículo No. 33. 1-25.

Cazorla, L. (2012). Presidente ejecutivo y gobierno corporativo de sociedades cotizadas en España: una aproximación al estado de la cuestión al hilo del reciente libro verde sobre gobierno corporativo de la Unión Europea. Departamento de Derecho Mercantil. Facultad de Derecho. Universidad Complutense. España.

Ciulla, J. B. 2014. Ethics, the Heart of Leadership. $3^{\text {rd }}$ edition. Editorial Praeger, Westport, USA.

Galve, C. (2002). Propiedad y gobierno: la empresa familiar. Revista Vasca de Economía Ekonomiaz. $\mathrm{N}^{\circ}$ 50. $2^{\circ}$ Cuatrimestre. 158-181.

Ganga, F. Abello, J. Quiroz, J. (2014). Gobernanza universitaria: una mirada histórica y conceptual. En: Ganga, F.; Abello, J.; Quiroz, J. Gobernanza universitaria, aproximaciones teóricas y empíricas. (pp. 11-20). Santiago: CEDAC, Universidad de Los Lagos, Santiago de Chile.

Ganga, F., Fossatti, P. \& Quiroz, J. (2016). A synchronic analysis of university governance: a theoretical view of the sixties and seventies. Revista Educação e Pesquisa 42(3), 1-16.

Goldspink, C. \& Kay R. 2009. Autopoiesis and organizations: A biological view of organizational change and methods for its study. In, Magalhaes, R. \& Sanchez, R. (eds), Autopoiesis in Organizations and Information Systems", Elsevier Science (Advanced Series in Management). 
Martínez, A. (2003). Dualidad de poder y rentabilidad de los accionistas: una explicación a través de la teoría de la agencia y la dirección estratégica. España: Universidad de León. XIII Jornadas Hispano-Lusas de Gestión Científica. 1-10. Maturana, H \& Varela, FJ. 1980. Autopoiesis and cognition: the realization of the living, BSPS, vol. 42, D. Reidel, Boston.

Mínguez, A. y J. Martín (2005). La influencia del poder de la dirección en el riesgo y el valor de la empresa: evidencia para el mercado español. España: Instituto Valenciano de Investigaciones Económicas, S.A. 1-26.

Olivencia (1998): El gobierno de las sociedades cotizadas. Comisión Especial para el Estudio de un Código Ético de los Consejos de Administración de las Sociedades.

Patton, A., y J. Baker. (1987): Why Won't Directors Rock the Boat? Harvard Business Review (November-December).

C.J. King. 2008. Ethics and leadership: reflections from a public research university. Center for Studies in Higher Education. University of California, Berkeley. Research \& Occasional Paper Series: CSHE.3.08 (March 2008).

Stein, G. y J. Capape (2009): Factores de fracaso del CEO: mapa de un debate. IESE Business School. Universidad de Navarra. ESTUDIO 85.

Termes, R. (1998): La reforma de los consejos de administración en España. Jornadas organizadas por la Asociación para el Progreso de la Dirección. Disponible en Internet: http://web.iese.edu/Rtermes/acer/acer13.htm University of Oxford, 2006. White Paper on University Governance. Oxford University Gazette, Vol. 136 Supplement 5 (May 2006). 
